\title{
DOSSIẾ
}

Sociologias, Porto Alegre, ano 4, № 7, jan/jun 2002, p. 136-155

\section{Arranjos de vida de idosos nos Estados Unidos*}

JAMET WIINOTH

\section{Razões para o estudo dos arranjos de vida para idosos}

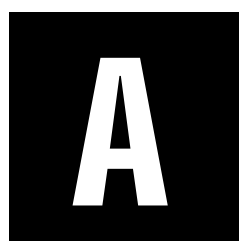

rranjos de vida para idosos - com quem e onde vivem são componentes críticos do envelhecer bem sucedido, porque eles determinam o ambiente físico e social onde ocorre 0 envelhecimento. Existem cinco tipos comuns de arranjos de vida para idosos nos Estados Unidos: o ancião mora sozinho; vive com um cônjuge; co-reside com filhos ou outra família; vive com pessoas sem laços de parentesco ou em uma instituição (ou seja, asilo, casa geriátrica). Cada um dos arranjos supracitados apresenta desafios singulares tanto para o idoso quanto para seus familiares e para a comunidade que os abrange. Q uestões específicas dependem do tipo de arranjo de vida para idosos a ser considerado. De todas as possibilidades de moradia e co-habitação, a vida com um cônjuge tende a ser a menos problemática, ao passo que a institucionalização é freqüentemente a mais problemática.

Ainda que viver com um cônjuge ou sozinho sejam arranjos de vida socialmente desejáveis para o idoso, é fato prontamente reconhecido que pessoas mais velhas, nestas situações, estão sob risco de precisar de assistência - particularmente aqueles que vivem sós. Idosos que vivem sozinhos precisam, muitas vezes, de assistência para várias atividades que vão desde tarefas doméstica rotineiras, (i.e. atividades instrumentais do viver diário), como limpar, preparar refeições ou cuidar do jardim, até cuidados pessoais (i.e. atividades físicas do dia-a-dia), como banhar-se ou alimen-

* Este artigo foi apresentado no IX Congresso Brasileiro de Sociologia, em Porto Alegre, 1999. Promoção da Sociedade Brasileira de Sociologia (SBS). A tradução do original em inglês é de autoria de Eurídice Baumgarten.

** Ph.D. e Professora Assistente de Sociologia/Purdue University, Indiana, EUA. E-mail: wilmothj@ sri.soc.purdue.edu 
tar-se. Família e amigos e/ou serviços comunitários formais encarregam-se da provisão desses serviços. Considerando que, nos Estados Unidos, todos os indivíduos de 60 anos ou mais são elegíveis para receber serviços comunitários, de acordo com o Título III da Lei dos Americanos Idosos (O Ider American's Act), o número de idosos que vivem sós e precisam de assistência é essencial para a formação de políticas governamentais e planejamento de programas.

Q uanto idosos que vivem sós estão sob risco de precisar de ajuda institucional depende do caráter de suas redes sociais. Pesquisa em redes de idosos apontam para uma hierarquia de dependência de outros, hierarquia na qual amigos e vizinhos preenchem necessidade básicas, diárias, e membros da família se encarregam do cuidado mais pessoal, de longo prazo. Mais serviços formais são usados quando suporte social informal é inacessível ou não-disponível (Krout, 1984; Litwark, 1985; Scott e Roberto, 1985). Os idosos que vivem sós estão particularmente sob risco de usar serviços formais (Avery, Speare at alii, 1989; Krout, Cutler et alii, 1990; Wilmoth, De Jong et alii, 1992). Ainda assim, apesar do maior risco de utilização de serviços, o ato de viver só é amplamente aceito como um fator positivo para o bem-estar do idoso, porque lhe permite manter-se independente. Em geral, é aceita a hipótese de que o impacto negativo potencial deste arranjo é amplamente compensado pela independência proporcionada pelo viver só.

A vida com outros, particularmente a co-residência com filhos, é vista como uma experiência problemática tanto para o idoso em questão como para seus filhos, já que põe pressão nas relações familiares. Esta noção de co-residência é largamente baseada nas normas culturais estadunidenses no que concerne a independência e individualismo. Coresidência é amiúde entendida como uma alternativa para um nível elevado de cuidado com os pais. Entretanto a pesquisa recente tem mostrado que são, com freqüência, as necessidades dos filhos e não as dos pais a definir a co-residência (Ward, Logan e Spitze, 1993). Além do mais, há 
evidências de que a co-residência não é uma experiência estressante, pelo menos para pais que vivem com filhos adultos jovens, de 19 a 34 anos de idade (Aquilino, 1991). Conseqüentemente, existe evidência limitada de que co-residência entre pessoas mais velhas e seus filhos adultos não é necessariamente em detrimento do bem-estar do idoso.

0 interesse sobre arranjos de coexistência (i.e., de vida) institucionais é influenciado por seu impacto, não só sobre os idosos em questão como também sobre suas famílias e até mesmo sobre a saúde fiscal do governo. 0 número de pessoas mais velhas que requerem institucionalização é uma séria preocupação para o governo, porque a assistência médica e social de longo prazo é financiada primariamente pelo Medicaid um programa financiado pelo Governo Federal dos Estados U nidos. Devido aos altos custos da assistência de longo prazo, a maioria dos indivíduos e famílias não têm condições de arcar sozinhos com estes serviços. 0 Medicaid tem 0 encargo de providenciar assistência financeira a indivíduos que não dispõem dos recursos financeiros para pagar por assistência de longo prazo. Daí que aumentos no número de idosos que carecem de cuidados extensivos por um longo período de tempo podem afetar negativamente os recursos governamentais.

Da perspectiva da família, colocar um pai ou outro parente idoso em uma instituição é um processo estressante. Apesar das normas culturais de independência e individualismo, há também, nos Estados U nidos, fortes normas quanto à provisão de cuidados a membros da família em dificuldades, sobretudo quando se trata de pai ou mãe. Internar os pais numa instituição viola essas normas, o que força a família a avaliar criticamente 0 seu curso de ação. Além disso, internar um pai ou mãe já fragilizados pela velhice, em um ambiente institucionalizado produz inquietações a respeito do impacto dessa internação no bem-estar da pessoa já em processo de envelhecimento. Há uma percepção de que o bem-estar do idoso tende a sofrer com este tipo de solução. Segundo as descobertas de Wolinsky et alii. (1992) sobre o impacto do internamento em casa geriátrica na morta- 
lidade dos velhos, parece que as preocupações de familiares a respeito do bem-estar de seus pais institucionalizados não são infundadas.

Como indica esta discussão prévia, é importante que examinemos os arranjos de coexistência dos idosos, por várias razões. A maior partes delas gira ao redor do bem-estar do idoso e sua família, assim com o da responsabilidade do governo na provisão de serviços comunitários e cuidados de longa duração para uma crescente população de velhos.

\section{O que se sabe sobre os arranjos de vida dos idosos nos Estados Unidos}

Devido à importância dos arranjos de vida para o bem-estar da população idosa, um setor substancial da pesquisa gerontológica nos Estados U nidos tem abordado este tópico. A maior parte dos pesquisadores têm assumido uma perspectiva estática, interseccional, na qual os arranjos de vida são explicados em termos de taxas agregadas em diferentes épocas e em termos dos vários fatores de nível individual que influenciam os arranjos de vida.

\section{Taxas agregadas e mudanças temporais nos arranjos de vida dosidosos estadunidenses}

Estudos interseccionais de arranjos de vida de idosos têm mostrado que a maioria (95\%) dos idosos estadunidenses vive em acomodações não institucionalizadas, ao passo que apenas $5 \%$ vivem em instituições a qualquer tempo na vida. Entre os idosos não institucionalizados, uma maioria (53\%) vive com um cônjuge. A seguir vêm os que vivem sós (30\%), com parentes (14\%) e com não-parentes (3\%) (Siegel, 1993). Ao longo do século XX, os arranjos de vida dos idosos transformaram-se substancialmente. A percentagem de velhos que vivem sós aumentou enquanto diminuiu a proporção daqueles que moram com parentes. Esta tendência para a vida 
independente é freqüentemente atribuída a preferências por viver só, que têm sido reconhecidas tanto no contexto dos progressos econômico e de estado de saúde dos idosos quanto através de mudanças em normas que concernem a arranjos de vida não-familiares (M ichael, Fuchs et alii, 1980; Pampel, 1983). Em geral, esta pesquisa baseada demograficamente providenciou informação descritiva sobre as tendências dos arranjos de vida dos idosos, de acordo com o período histórico.

\section{Características de nível individual relacionadas aos arranjos de vida estadunidenses}

Dois distintos, ainda que compatíveis, modelos conceituais emergiram da literatura sociológica e gerontológica para explicar os efeitos das características de nível individual em arranjos de vida de idosos. 0 primeiro é um modelo doméstico de tomadas de decisão que contém quatro categorias de variáveis que identificam os mecanismos fundamentais que motivam os arranjos de vida dos idosos. Entre essas categorias estão oportunidades, recursos, necessidades e preferências (W olf e Soldo, 1988; Spitze, Logan et alii, 1992). 0 segundo modelo, que inclui fatores de disponibilidade, viabilidade e desejo, foi originalmente desenvolvido para explicar variações nacionais de idade na data do casamento e proporção de nunca casados (Dixon, 1971). Burr e Mutchler (1992) expandiram esses fatores para explicar diferenças nos arranjos de vida entre diferentes populações étnicas e raciais no território dos Estados Unidos.

Sejam estes modelos usados para explicar tomadas de decisão domésticas, relativas aos arranjos de vida dos idosos, ou diferenças nestes arranjos de vida através de subpopulações, as variáveis são praticamente idênticas. A categoria oportunidade, que inclui variáveis como estrutura de família, corresponde diretamente a fatores de disponibilidade. Recursos e necessidades, incluindo recursos econômicos, saúde, e deficiência (física ou mental), estão incluídos no fator de viabilidade. A categoria pre- 
ferência é análoga ao fator desejo, se considerarmos que ambas as categorias consistem de variáveis que indiretamente medem a percepção individual de arranjos de vida os melhores possíveis. No nível mais básico possível, estes dois modelos são tentativas de capturar as variáveis fundamentais que influenciam os arranjos de vida de idosos: estrutura familiar, recursos econômicos, saúde e características demográficas individuais dos idosos em questão.

\section{Fatores de oportunidade e disponibilidade}

A categoria disponibilidade/oportunidade inclui características da estrutura da família do idoso e relacionamentos entre diferentes gerações, que podem constranger escolhas de arranjos de vida. A maior parte da pesquisa em arranjos de vida de idosos que inclui medidas de estrutura de família geralmente enfoca as características dos filhos do idoso, em particular o número e gênero dos filhos. 0 número de filhos é usado como uma medida do grupo potencial de pessoas envolvidas no cuidado do idoso e/ ou filhos com quem co-habitar. Considerando que as mulheres são, geralmente, quem toma a responsabilidade de cuidar dos idosos, ter uma filha mulher aumenta as chances de receber ajuda e viver com os filhos (Soldo, Wolf et alii, 1990; Stone, Cafferata e Sangl, 1987). De maneira geral, o número de filhos é negativamente relacionado à possibilidade de viver só; idosos sem filhos ou com um único filho são significativamente mais passíveis de viverem sozinhos (Avery et alii. 1989; Soldo et alii, 1990). Mulheres com filhas apenas, têm mais possibilidade de viver com uma filha que mulheres que têm apenas filhos, ao passo que mulheres com filhos de ambos os sexos têm probabilidades intermediárias de viver com um dos filhos (Soldo et alii, 1990).

Ainda que filhos, e particularmente os do sexo feminino, aumentem as chances de co-residência, há evidência de que os filhos podem oferecer apoio aos seus velhos pais, que possibilite aos mesmos manter a sua pró- 
pria residência independente. Idosos que têm mais filhos disponíveis têm mais chance de viver sós, apenas com o apoio de ajudantes informais (Soldo et alii, 1990). Deve-se notar que a co-residência com filhos adultos não se dá apenas em função das necessidades dos pais. Pesquisas recentes sugerem que as necessidades dos filhos adultos são com freqüência tão importantes, senão mais, no processo de determinação de co-residência, do que as necessidades características dos pais.

Além dessas noções básicas das características dos filhos, pouco se sabe sobre como outras particularidades de filhos adultos (i.e. estado civil, colocação no mercado de trabalho, número de filhos, proximidade com os velhos pais, etc.) influenciam os arranjos de vida dos idosos. Pesquisas sugerem que as características dos filhos adultos de fato têm um impacto em arranjos de vida de idosos, especialmente co-residência. Filhos solteiros, especialmente do sexo masculino, são significativamente mais passíveis de co-residir, ao passo que filhas casadas que estão no mercado de trabalho têm menos probabilidade de morar com os pais (W olf e Soldo, 1988). Há também evidência de que o número de filhos não é, por si só, eficiente para prever co-residência, uma vez que usemos como parâmetro o estado civil dos filhos (Crimmins e Ingegneri, 1990). Tirando-se esses estudos, o efeito das características dos fiIhos adultos em arranjos de vida não co-residenciais não foi ainda cuidadosamente examinado. Além do mais, não se tem ainda clara a maneira como relacionamentos entre gerações influenciam arranjos de vida de idosos. Sobretudo, há mais para ser aprendido sobre a influência da estrutura familiar e relacionamentos entre gerações nos arranjos de vida de idosos.

\section{Fatores de viabilidade/recursos e necessidades}

Fatores de viabilidade incluem quaisquer características que obstruam ou facilitem específicos arranjos de vida. Essas características são geralmente relacionadas aos recursos econômicos e de saúde do idoso, que influenciam suas necessidades por apoio físico, instrumental ou emocio- 
nal. A idade cronológica é muitas vezes usada em análises de arranjos de vida como um substituto do processo de envelhecimento e dos eventos da vida que acompanham o envelhecer. À medida que a idade aumenta, a proporção de idosos morando com um cônjuge declina, ao passo que a proporção dos que vivem sós, em co-residência e em instituições cresce (Litwak e Longino, 1987; Siegal, 1993). Estas tendências são particularmente exageradas para mulheres. Estas apresentam menos probabilidade do que os homens de estarem vivendo com um cônjuge e são mais suscetíveis de estarem vivendo sozinhas, a qualquer idade (Siegal, 1993). Isso se deve largamente à diferença de mortalidade entre homens e mulheres. Entretanto é importante notar que são eventos da vida, tais como viuvez, doença e deficiência, física ou mental, que influenciam diretamente arranjos de vida e não, a idade cronológica. Por exemplo, Crimmins e Ingegneri (1990) concluíram que a idade não é significante quando da previsão de co-residência, uma vez que trabalhemos com os parâmetros de viuvez e deficiência. Conseqüentemente a viabilidade de um arranjo de vida é influenciada pelos eventos da vida que ocorrem durante o processo de envelhecimento, porque esses eventos têm um efeito sobre os recursos econômicos e de saúde do idoso.

Arranjos de vida de idosos são diretamente ligados ao estado civil, já que a maioria dos velhos que são casados vive com o respectivo cônjuge. Em função disso, muitos estudos que examinam arranjos de vida de idosos se concentram apenas nos respondentes não casados - ou seja, nunca casados, divorciados/separados ou viúvos - (e.g. Burr e Mutchler, 1992; Soldo e Wolf, 1988; Spitze et alii, 1992; Worobey e Angel, 1990b). Freqüentemente uma medida do estado civil que atua, de forma indireta, como parâmetro da história matrimonial é incluído nessas análises, mas pouco se sabe acerca do impacto da viuvez nos arranjos de vida. O bviamente, a experiência da morte de um cônjuge demanda uma mudança nos arranjos de vida do sobrevivente, mas como essa experiência influencia as transições do arranjos de vida subseqüentes? 
Há alguma evidência de que metade das viúvas continua a viver no mesmo local depois da morte do esposo, e que, entre aquelas que mudam de residência depois da viuvez 39\%, fazem-no no prazo de seis meses. Além do mais, a maioria dessas viúvas mora em sua própria casa (70\%), sozinhas (50\%) (Lopata, 1973). Ainda assim, não sabemos se o processo das viúvas para arranjarem a nova vida difere significativamente da experiência de indivíduos que nunca foram casados, divorciados ou separados, ou como a idade ao tempo em que ocorre a viuvez influencia o processo. Então existem aspectos que precisam ser melhor explorados, aspectos esses relacionados ao impacto da viuvez em arranjos de vida de homens e mulheres idosos.

Arranjos de vida e saúde estão estreitamente relacionados. Saúde declinante e crescente deficiência (física, mental) diminuem as chances que uma pessoa idosa teria de viver sozinha, ao mesmo tempo em que aumentam a possibilidade de essa pessoa morar com outros ou ser internada (Angel, 1991; Avery et alii, 1989; Mutchler e Burr, 1991; W orobey e Angel, 1990b). Curiosamente, melhorias na capacidade funcional, com 0 passar do tempo, suprimem a necessidade de mudanças nos arranjos de vida, mesmo para os indivíduos com baixos níveis iniciais de capacidade funcional. Idosos que experimentam progressos nas atividades instrumentais do viver diário (IADL, do inglês Instrumental Activities of Daily Living) têm a menor probabilidade de fazer ajustamentos no seu ambiente de vivência (definido como uma mudança geográfica e/ou uma alteração nos arranjos de vida), enquanto idosos que têm o maior declínio das funções de IADL têm a mais alta possibilidade de experimentar uma mudança no seu ambiente de vivência (Jackson, Longino et alii, 1991). Conclui-se que são as mudanças na saúde e deficiência, e não níveis absolutos, que têm a maior influência em transições de arranjos de vida.

Um último fator que influencia a viabilidade dos arranjos de vida de idosos é a situação financeira. É amplamente aceito que recursos econômicos facilitam arranjos de vida independente, porque esses recursos provêem os meios para a escolha do local de moradia preferido pelo idoso, 
assim como a obtenção de serviços que possibilitem a manutenção de um modo de vida independente. Por exemplo, dentro dos parâmetros de deficiências de saúde e de disponibilidade de filhos, mulheres idosas com suficientes recursos têm uma tendência a morar sozinhas. Estas descobertas sugerem que mulheres idosas preferem viver sós, já que aquelas que dispunham dos meios financeiros para tal moravam sós, ainda que sofressem de saúde deficiente (Soldo et alii, 1990). Pesquisa anterior concluiu que independência financeira, ou a falta dela são determinantes mais importantes nos arranjos de vida, (particularmente os que implicam em morar com outros), dos idosos negros ou hispânicos do que entre os brancos (Worobey e Angel, 1990a). Recursos financeiros são, então, fatores determinantes dos arranjos de vida para idosos estadunidenses.

\section{Fatores de desejo e preferência}

Finalmente, fatores de desejo incluem preferências individuais por tipos específicos de arranjos de vida. Já que preferências em arranjos de vida não são, em geral, medidas diretamente, orientação cultural (medida indiretamente, através de auto-referência do público alvo) é usada como um substituto para preferência e desejo. O rientações culturais que defendem o familismo, um sistema de valores no qual as necessidades da família suplantam as dos indivíduos que nela tomam parte, promovem a co-residência e lares onde convivem múltiplas gerações de uma mesma família. Pesquisa sobre a população mais velha nos Estados Unidos, que inclui medidas de raça/etnia e condição do imigrante indica; 1 ) idosos não-brancos têm significativamente mais possibilidade de estar morando com outros (freqüentemente como chefe da família), do que idosos brancos; 2) a formação de lares não-brancos, nos quais convivem famílias extensivas ${ }^{1}$, ocorre devido à desvantagem econômica e não, ao mau estado de saúde do idoso; 3) há uma variação considerável em termos de arranjos de vida

1 A família norte-americana de origem anglo-saxônica (EUA e Canadá), compõe-se quase que exclusivamente do mais restrito núcleo familiar: pai, mãe, um ou dois filhos jovens. Sobrinhos, primos, avós, tios, cunhados, etc. fazem parte do que nestes países é chamado de família extensiva, um tipo de organização familiar presente na vida dos imigrantes denominados "não-brancos", ou seja, latino-americanos, asiáticos, africanos... (N. do T.). 
dos idosos, conforme os parâmetros de grupos étnicos e raciais e 4) imigrantes recentes são significativamente mais suscetíveis de estar morando com outros do que os seus semelhantes étnicos e raciais (Burr e M utchler, 1992a, 1993; Worobey e Angel, 1990a; Himes, Hogan et alii, 1996; Wilmoth, De Jong et alii, 1997). Estas diferenças observadas entre raças/ grupos étnicos são interpretadas como evidência de preferências culturais subjacentes por certos tipos de arranjos de vida na velhice.

Contudo existe alguma evidência de que essas preferências culturais são mediadas por outros fatores, que, quando incluídos na análise, minimizam ou eliminam o efeito significante das variáveis de raça/etnia. Um desses fatores é o conjunto de características dos filhos adultos do idoso. Se o parâmetro de controle for o estado civil dos filhos, reduz-se a diferença nas taxas de coresidência de idosos negros e brancos (Aquilino, 1990; Crimmins e Ingegneri, 1990). O utro fator é a renda, que diminui os efeitos das preferências culturais nos arranjos de vida de idosos (Burr e Mutchler, 1993).

Portanto, a pesquisa prévia mostra que raça e etnia podem servir como substitutos às preferências de arranjos de vida, mas como qualquer substituto, estas variáveis podem incluir os efeitos de variáveis não mensuradas. Por isso, é importante trabalhar com modelos corretamente especificados e considerar o impacto potencial de variáveis não mensuradas em variáveis substitutas.

Finalmente, educação pode influir diretamente nos arranjos de vida dos idosos, na medida em que forma percepções individuais a respeito do que é desejável em determinados arranjos de vida, ou indiretamente, através de seu impacto em outros fatores, como saúde e número de filhos, que são determinantes importantes em arranjos de vida. Enquanto o impacto da educação na percepção daquilo que é desejável em específicos arranjos de vida não foi minuciosamente examinado, há alguma evidência quanto ao seu efeito indireto sobre arranjos de vida na velhice. Em geral, idosos com altos níveis de educação são mais passíveis de viverem sós e menos de coresidir com filhos (Avery et alii, 1989; Crimmins e Ingegneri, 1990). Crimmins e Ingegneri (1990), atribuem esta tendência ao melhor estado de saúde e menor número de filhos de idosos com educação mais elevada. 


\section{Transições em arranjos de vida entre idosos nos Estados U nidos}

A pesquisa sobre arranjos de vida de idosos nos Estados U nidos progrediu, ao longo dos últimos trinta anos, de estudos interseccionais que documentam as tendências demográficas, para análises interseccionais e longitudinais dos determinantes de nível individual dos arranjos de vida .M ais recentemente, arranjos de vida têm sido analisados sob uma perspectiva dinâmica que focaliza o processo de transição (W ilmoth, 1998; W ilmoth, no prelo). Essa pesquisa conceitualiza transições em arranjos de vida como uma adaptação ao processo de envelhecimento, que é necessário para a manutenção da competência. De acordo com Lawton (1982), a habilidade de um indivíduo para funcionar efetivamente em um determinado ambiente é o produto da competência do indivíduo, somada à pressão ambiental. Diversos eventos podem influenciar o nível de competência de um indivíduo ou alterar o nível da pressão ambiental, em qualquer das hipóteses forçando o indivíduo a se adaptar à nova situação. Este ajustamento pode variar, de um processo adaptativo a um mal-adaptativo, o que dependerá da capacidade de reserva do indivíduo.

Q uando se aplicam esses conceitos às transições em arranjos de vida, o processo de envelhecimento inclui vários eventos, tais como aposentadoria, decréscimo de renda, saúde falha e morte de um cônjuge, eventos estes que alteram a habilidade da pessoa em processo de envelhecimento a suportar o seu ambiente e/ou aumentam a pressão ambiental. Alguns desses eventos, tais como a morte de um cônjuge, causam uma mudança imediata em arranjos de vida, ao passo que outros, como saúde em declínio, forçam 0 idoso a reconsiderar a propriedade de seus arranjos de vida. Em qualquer desses casos, adaptações permitem ao idoso alterar o seu ambiente físico e pessoal (i.e. suporte social), de tal maneira que a habilidade do idoso para funcionar efetivamente é aperfeiçoada. Estas adaptações podem tomar várias formas, até mesmo a alteração da estrutura física do espaço de vivência, mudança da composição do lar ou a transferência para um novo ambiente. O bservadas desta perspectiva pessoa/ambiente, transições em arranjos de 
vida podem ser entendidas como o resultado de eventos que ocorrem no curso da vida, durante o envelhecimento, cuja influência é mediada pelas características do idoso e suas redes de suporte social.

A figura 1, que mostra o espaço do estado das transições em arranjos de vida, apresenta quatro estados de arranjos de vida que o idoso pode experienciar e os diversos caminhos através do quais os seus arranjos de vida podem mudar. Para simplificar o modelo, arranjos de vida que envolvem viver com filhos, parentes, e/ou não-parentes foram incluídos na categoria de co-residência. Ao longo do tempo, os indivíduos movem-se entre esses diferentes estados de arranjos de vida, criando uma história de transições em arranjos de vida (ou não-transições, para casos nos quais não ocorrem quaisquer mudanças) Essa história de transições é de primário interesse para essa área de pesquisa emergente. Especificamente, a que extensão ocorrem as transições entre arranjos de vida e como são estas relacionadas à idade?

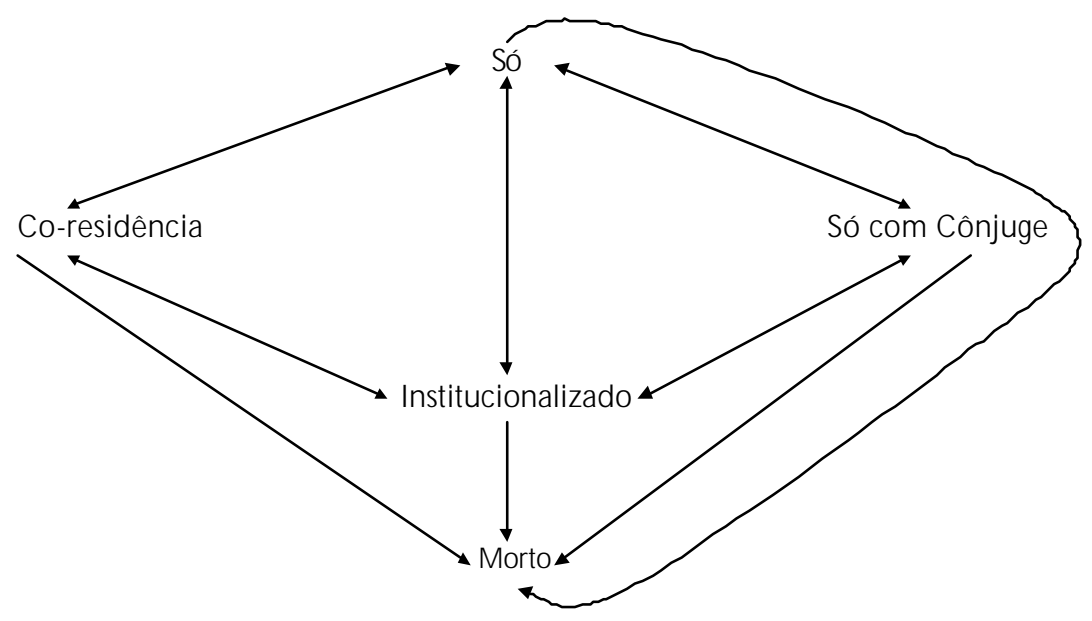

Figura 1. Transições em Arranjos de Vida 
Para responder a essas questões, Wilmoth (1998) usa métodos da história de eventos para analisar os dados da primeira e segunda ondas do Levantamento Nacional de Famílias e Unidades Domésticas (NSFH, do inglês National Survey of Families and Households). O NSFH de 19871988 contém uma amostra representativa nacional de 13.017 entrevistados de 19 anos de idade ou mais, vivendo em unidades domésticas nos Estados U nidos. A primeira amostra do NSFH foi seguida em 1993-1994. Ao todo, 3.339 respondentes, cuja idade era de 55 anos ou mais na primeira entrevista foram re-entrevistados. Também foram incluídas na análise pessoas nas idades de 50 a 54 anos que completaram 55 entre a primeira e a segunda entrevista. Apesar de a maior parte dos estudos de arranjos de vida de idosos nos Estados Unidos usarem amostras de 65 anos e acima, expandir a variação de idade para acima de 55 habilita essa pesquisa a lidar com problemas da transição entre a população na idade pré-aposentadoria e recém-aposentada.

Estes achados, que são detalhadamente referidos por W ilmoth (1998), indicam que a probabilidade de se fazer uma transição de arranjo de vida aumenta com a idade, embora isso varie entre tipos diferentes de transição. Antes dos 70 anos, os mais estáveis arranjos de vida são os que implicam em morar só ou com um cônjuge. A maior parte das pessoas que faz transições neste período inicial do envelhecimento está, ou trocando de estado civil, ou tem um filho deixando a casa ou mudando-se para ela. Depois dos 70 anos, o arranjo de vida mais estável é morar com um filho. A maior parte das transições depois dessa idade é devida à institucionalização e morte. Viver com um cônjuge e filhos é particularmente instável, devido ao maior número de pessoas que pode mudar-se para a casa ou para fora dela. Essa pesquisa revela, sobretudo, a natureza dinâmica dos arranj0os de vida para idosos nos Estados U nidos. 


\section{Caminhos para pesquisas futuras}

Ao passo que essa recente análise encoraja pesquisadores a considerarem a natureza dinâmica dos arranjos de vida na terceira idade, há ainda muitas questões que precisam ser trabalhadas. Por exemplo, essa pesquisa recente examina apenas a primeira transição de arranjo de vida. $\mathrm{Nem}$ as transições subseqüentes nem o processo completo de transição são aqui considerados. Devido a limitações nos dados, transições envolvendo outros (e.g. netos e não-parentes), não puderam ser examinados. Além disso, essa pesquisa recente não leva em consideração o modo como características de indivíduos, famílias e comunidades afetam o processo de transição. Wilmoth (no prelo) explora os efeitos de câmbios sociais informais sobre transições em arranjos de vida, mas há mais para ser descoberto a respeito da influência da utilização de serviço formal.

Finalmente estamos apenas começando a entender os impactos, a curto e longo-prazo, das transições de arranjos de vida no bem-estar dos idosos. A maior parte do que se sabe é baseado na literatura gerontológica que examina os resultados da institucionalização. Em geral, a pesquisa sobre os resultados da institucionalização no bem-estar indica que esta tem um efeito negativo na saúde, embora diversas características individuais e institucionais intermedeiem essa relação (Baglioni, 1989; Coffman, 1983; Engel e Graney, 1993; Wolinsky, Callahan et alii, 1992). Porém a maior limitação desses estudos é que eles, muitas vezes, enfocam apenas residentes de institutos geriátricos e não os comparam com os seus semeIhantes que permanecem na comunidade (ou retornam à comunidade depois de um período no hospital ou casa geriátrica).

Há apenas uns poucos estudos que exploram os resultados do bemestar em arranjos de vida entre a comunidade dos idosos (Ferraro, 1982; Lawton, M oss et alii, 1984; Magaziner, Cadigan et alii, 1988; Davis, N euhaus et alii, 1992). Em geral, os resultados desses estudos são mistos; viver só tem resultados tanto negativos como positivos. Não obstante, esses resultados são passíveis de descrever inadequadamente a relação entre transi- 
ções em arranjos de vida e bem-estar, já que a maioria dos estudos se ocupa de mulheres idosas e de dados interseccionais. Portanto, há uma necessidade de examinar os resultados do bem-estar dos diversos arranjos de vida de idosos usando dados longitudinais.

Concluindo, uma quantidade considerável de pesquisa sobre arranjos de vida de idosos tem sido publicada nos Estados Unidos nos últimos 30 anos. Essa pesquisa identificou a distribuição dos arranjos de vida entre as populações idosas e os fatores de nível individual que influenciam esses arranjos de vida. Dados longitudinais estão sendo cada vez mais usados para modelar mudanças individuais em arranjos de vida no curso do tempo. Ainda que essa pesquisa recente tenha demonstrado que arranjos de vida para a terceira idade podem ser encarados como um processo dinâmico, há mais a ser conhecido sobre esse processo. A pesquisa futura, que usa uma perspectiva dinâmica no estudo dos processos das transições em arranjos de vida contribuirá para o nosso entendimento dos contextos físico e social do enveIhecer, o que assistirá provedores de serviços no planejamento das necessidades da crescente população de adultos velhos nos Estados U nidos.

\section{Referências bibliográficas}

ANGEL, Jacqueline. Health and living arrangements of the elderly. New York: Garland Publishing, 1991.

AQ UILIN O, W illiam S. The likelihood of parent-adult child co-residence: effects of family structure and parental characteristics. Journal of M arriage and the Family 52,1990, p. 405-419.

AQ UILIN O, W illiam S. Parent-child relations and parent's satisfaction with living arrangements when adult children live at home. Journal of M arriage and the Family 53, 1991, p.13-27.

AVERY, R.; SPEARE, Alden; et alii. Social support, disability and independent living of elderly persons in the U nited States. Journal of Aging Studies 3, 1989, p. 279-293.

BAGLION I. A. Residential relocation and health of the elderly. In: K.S. M arkides and C.L. Cooper (eds.)Aging, Stress, and Health. New York: John, W iley, and Sons, 1989. 
BURR, Jeffery e MUTCHLER, Jan. The living arrangements of unmarried elderly hispanic females. 1992.

BURR, Jeffery e MUTCHLER, Jan. Nativity, acculturation, and economic status: explanations of asian american living arrangements in later life. Journals of Gerontology: Social Sciences 48, 1993a, p.S55-63.

BURR, Jeffery e MUTCH LER, Jan. Ethnic living arrangements: cultural convergence or cultural manifestation. Social Forces 72, 1993b, p.169-179.

COFFM AN, T. Toward and understanding of geriatric relocation. The Gerontologist 23, 1983, p.453-459.

CRIMM INS, Eileen M. e INGEGNERI, Dominique G. Interaction and living arrangements of older parents and their children: Past trends, present determinants, future implications. Research on Aging 12, 1990, p.3-35.

DAVIS, Maradee A.; NEUHAUS, John M.; et alii. Living arrangements and survival among middle-aged and older adults in the NH ANESI Epidemiologic Follow-up Study. American Journal of Public Health 82, 1992, p.401-406.

DIXON, Ruth B. Explaining cross-cultural variations in age at marriage and proportions never marrying. Population Studies 25, 1971, p.215-233.

ENGEL, Veronica F. e GRANEY, Marshall J. Stability and improvement of health after nursing home admission. Journalsof Gerontology: Social Sciences 48, 1993, p.17-23.

FERRARO, $\mathrm{K}$. The Health consequences of relocation among the aged in the community. Journals of Gerontology 38, 1982, p.90-96.

HIMES, C. L.; HO GAN, D. P.; et alii. Living arrangements of minority elders. Journal of Gerontology: Social Sciences51B, 1996, p.42-48.

JACKSO N, David J.; LO NGINO, Charles F.; et alii. Environmental adjustments to declining functional ability: Residential mobility and living arrangements. Research on Aging 13, 1991, p.289-309.

KROUT, John A. Utilization of services by the elderly. Social Services Review 58, 1984, p.281-290. 
KROUT, John A.; CULTER, Stephen J.; et alii. Correlates of senior center participation: a national analysis. The Gerontologist 30, 1990, p.72-79.

LAWTO N, M. Powell. Competence, environmental press, and the adaptation of older people. In: M. Powell Lawton, Paul G. W indley et alii (eds.) Aging and the environment: theoretical approaches. N ew York: Springer Publishing Co, 1982.

LAWTO N, M. Powell; MOSS, M iriam; et alii. Marital status, living arrangements, and the well-being of older people. Research on Aging 3, 1984, p.323-345.

LITW AK, E. H elping the elderly: the complementary role of informal networks and formal care systems. New York: Guildford, 1985.

LITWAK, E. e LONGINO, Charles F. Migration patterns among the elderly: a developmental perspective. The Gerontologist 27, 1986, p.266-274.

LO PATA, Helena Z. W idowhood in an American City. Cambridge, M A: Schenkman Publishing Co., 1973.

MAGAZINER, Jay; CADIGAN, D orisA; et alii. H ealth and living arrangementsamong older women: does living alone increase the risk of illness? Journal of Gerontology: Medical Sciences43, 1988, p.127-133.

MICHAEL, R.; FUCHS, V.; et alii. Changes in the propensity to live alone: 19501976. Demography 17, 1980, p.39-56.

MUTCHLER, Jan e BURR, Jeffery. A longitudinal analysis of household and nonhousehold living arrangements in later life. Demography 28, 1991, p.375-390.

PAM PEL, F. Changes in the propensity to live alone: Evidence from consecutive cross-sectional surveys, 1960-1976. Demography 20, 1983, p.433-447.

SCOTT, Jean Pearson e RO BERTO, Karen A. U se of informal and formal support networks by rural elderly poor. The Gerontologist 20, 1985, p.200-207.

SIEGEL, Jacob. A generation of change: a profile of America's O Ider Population. New York: Russell Sage Foundation, 1993.

SO LDO , Beth; W O LF, Douglas; et alii. Family, households, and care arrangements of frail older women: a structural analysis. Journal of Gerontology:Social Sciences 45,1990, p. $238-249$. 
SPITZE, Glenna; LOGAN, John; et alii. Family structure and changes in living arrangements among elderly non-married parents. Journals of Gerontology: Social Sciences 47, 1992, p.289-296.

STO NE, R., CAFFERATA, G.; et alii. Caregivers of the frail elderly: a national profile. The Gerontologist 27, 1987, p.616-626.

W ARD, Russell; LO GAN, John e SPITZE, Glenna. The influence of parent and child need on coresidence in middle and later life. Journal of $M$ arriage and the Family 55,1993, p. 209-221.

W ILM OTH, Janet M.; DE JO NG, Gordon F.; et alii. D eterminants of communitybased and in-home service utilization. Paper presented at the 1992 American Sociological Association Annual Meeting, W ashington, D.C, 1992.

W ILM OTH, Janet M.; DE JO NG, Gordon F.; et alii. Immigrant and N on-Immigrant Living Arrangements among America's White, Hispanic, and Asian Elderly Population. International Journal of Sociology and Social Policy 17, 1997, p.57-82.

W ILM O TH, Janet. Living arrangement transitions among America's O Ider Adults. The Gerontologist 38, 1998, p.434-444.

WILM OTH, Janet. U nbalanced social exchanges and living arrangement: transitions in later life, no prelo.

W O LF, D ouglas e SO LDO, Beth. Household composition choices of older unmarried women. Demography 25, 1988, p.387-403.

W OLINSKY, Fredric D; CALLAHAN, Christopher M.; et alii. The risk of nursing home placement and subsequent death among older adults. Journals of Gerontology: Social Sciences 47, 1992, p.173-182.

WO RO BEY, Jacqueline e ANGEL, Ronald. Poverty and health: older minority women and the rise of the female-headed household. Journal of $\mathrm{H}$ ealth and Social Behavior 31, 1990a., p.370-383.

WO ROBEY, Jacqueline e ANGEL, Ronald. Functional capacity and living arrangements of unmarried elderly persons. Journals of Gerontology: Social Sciences 45, 1990b, p.95-101. 
Sociologias, Porto Alegre, ano 4, no 7, jan/jun 2002, p. 136-155

\section{Resumo}

Este artigo repassa a literatura corrente no que se refere a arranjos de vida (condições e locais de moradia e co-habitação) entre idosos nos EU A. As razões para o estudo dos arranjos de vida estão delineadas e as tendências agregadas estão humanizadas. $0 \mathrm{~s}$ dois modelos conceituais comumente usados nesta área de pesquisa estão apresentados. Estes modelos identificam as características de nível que influenciam os arranjos de vida na terceira idade. Argumentamos que uma pesquisa mais dinâmica é necessária para explicar o processo de transição do arranjo de vida. Esta perspectiva se baseia na teoria de Lawton, que analisa a pessoa com seu próprio ambiente social e cultural e se baseia em uma análise longitudinal. Para terminar, discutimos os caminhos para a pesquisa futura.

Palavras-chave: arranjos de vida, composição doméstica do lar, transições de tipo de moradia. 